\title{
A Multi-Mode Sensing System for Corrosion Detection Using Piezoelectric Wafer Active Sensors
}

\author{
Lingyu $\mathrm{Yu}$, \\ Mechanical Engineering Department, University of South Carolina \\ Columbia, SC 29208, yu3@engr.sc.edu \\ Victor Giurgiutiu, \\ Mechanical Engineering Department, University of South Carolina \\ Columbia, SC 29208, giurgiut@engr.sc.edu \\ Patrick POLLOCK, \\ Mechanical Engineering Department, University of South Carolina \\ Columbia, SC 29208, pollocpj@engr.sc.edu
}

\begin{abstract}
As an emerging technology for in-situ damage detection and nondestructive evaluation, structural health monitoring with active sensors (active SHM) plays as a promising candidate for the pipeline inspection and diagnosis. Piezoelectric wafer active sensor (PWAS), as an active sensing device, can be permanently attached to the structure to interrogate it at will and can operate in propagating wave mode or electromechanical impedance mode. Its small size and low cost (about $\sim \$ 10$ each) make itself a potential and unique technology for in-situ SHM application. The objective of the research in this paper is to develop a permanently installed in-situ "multi-mode" sensing system for the corrosion monitoring and prediction of critical pipeline systems. Such a system is used during in-service period, recording and monitoring the changes of the pipelines over time, such as corrosion, wall thickness, etc. Having the real-time data available, maintenance strategies based on these data can then be developed to ensure a safe and less expensive operation of the pipeline systems.

After a detailed review of PWAS SHM methods, including ultrasonic, impedance, and thickness measurement, we introduce the concept of PWAS-based multi-mode sensing approach for corrosion detection in pipelines. Particularly, we investigate the potential for using PWAS waves for in thickness mode experimentally. Finally, experiments are conducted to verify the corrosion detection ability of the PWAS network in both metallic plate and pipe in a laboratory setting. Results show successful corrosion localization in both tests.
\end{abstract}

Keywords: corrosion detection, thickness, in-situ monitoring, piezoelectric wafer active sensor, PWAS, Lamb waves

\section{INTRODUCTION}

Pipelines are important civil infrastructures utilized to transport water, gas, petroleum, solvent or mixtures. They are typically subjected to corrosion along the inside of the pipe. Over time, the corrosion makes the pipe wall thinner and leaves the pipes vulnerable to failure. Failure of pipes and tubular equipment such as in petroleum plants tend to be catastrophic, resulting in costly property damage, loss of business continuity and even fatalities. There is an ever-growing global awareness of this issue.

Currently, there are two categories of commercially available solutions, either internal or external inspection. Internal inspection of tubes has been traditionally accomplished using a rotating transducer to scan the tube wall point by point [1][2]. External inspection of piping systems can be accomplished by a series of point tests performed from the outside of the pipe. If insulation covers the pipe, as it is often the case, access to the outside surface of the pipe requires removal of the insulation to perform the test, and then re-installing the insulation after the tests are complete. Ultrasonic techniques also permit quick and reliable measurement of thickness without requiring access to both sides of a part. The measurement is usually done by coupling the sound energy into the test piece through a water column generated by a bubbler or squirter, or in a water bath. The main drawbacks of conventional ultrasonic inspection techniques are they are point-wise inspection methods, laborintensive, requiring highly trained operators, and are nearly impossible to use for in-situ high-temperature SHM. Considering the limitations of conventional ultrasonic transducers, guided Lamb waves offer new opportunities for cost effective detection of damage in structures. The use of Lamb waves to detect the corrosion in aluminum structures using the pitch-catch method has been explored by Chahbaz et al [3][4]. Alleyne et al detected corrosion in pipes using the pulse-echo method using the mode conversion method [5]. Zhu and Rose conducted 
experimental study on hidden corrosion detection by using the ultrasonic guided waves combined with BEM numerical simulation [6].

This paper presents an in-situ multi-mode diagnostics/monitoring of critical pipeline system utilizing embedded piezoelectric wafer active sensors (PWAS) guided waves. After the sensor network is installed, three nondestructive methods including guided waves pitch-catch method, impedance method, and thickness measurement are used simply by changing the PWAS driving frequency. The corrosion damage is detected and quantified using the multiple methods. The multi-mode sensing was first verified on corrosion detection on aluminum plate and then repeated on pipe structure. Experimental results show that this method is effective in locating the corrosion damage while improvement is expected on the direct thickness measurement.

\section{PWAS NONDESTRUCTIVE METHODS}

\subsection{Piezoelectric Wafer Active Sensors}

PWAS are small, noninvasive, and inexpensive elastic wave generators/receptors that can be easily affixed to the structure and intimately connected into interrogative sensor arrays [7]. They can be wired to data concentrators and wireless communicators. PWAS have captured the interest of the academic and industrial community due to their low cost and small nonintrusive nature [8]-[10]. PWAS operate on the piezoelectric principle that couples the electrical and mechanical variables (mechanical strain $S_{i j}$, mechanical stress $T_{k l}$, electrical field $E_{k}$, and electrical displacement $D_{j}$ ) in the material in the form

$$
\begin{gathered}
S_{i j}=s_{i j k l}^{E} T_{k l}+d_{k i j} E_{k} \\
D_{j}=d_{j k l} T_{k l}+\varepsilon_{j k}^{T} E_{k}
\end{gathered}
$$

where $s_{i j k l}^{E}$ is the mechanical compliance of the material at zero electrical field $(E=0)$, T1 is the stress, $\varepsilon_{j k}^{T}$ is the dielectric constant at zero stress $(T=0)$, and $d_{k i j}$ is the induced strain coefficient (mechanical strain per unit electric field). PWAS utilize the $d_{31}$ coupling between in-plane strain and transverse electric field.

For a piezoelectric wafer as depicted in Figure 1, an electric field $E_{3}$ is applied parallel to the spontaneous polarization $P_{\mathrm{s}}$. If Ps is aligned with the $x_{3}$ axis, then the application of $E_{3}$ is created by applying a voltage $V$ between the bottom and top electrodes as shown in the Figure 1. The situation of $E_{3} / / P_{\mathrm{s}}$ results in a vertical (thickness wise) expansion $\varepsilon_{3}=d_{33} E_{3}$ and a lateral (in plane) contractions $\varepsilon_{1}=d_{31} E_{3}$ and $\varepsilon_{2}=d_{32} E_{3}$. The strains experienced by PWAS are direct strains and such an arrangement can be used to produce thickness-wise and inplane vibration of PWAS [11].

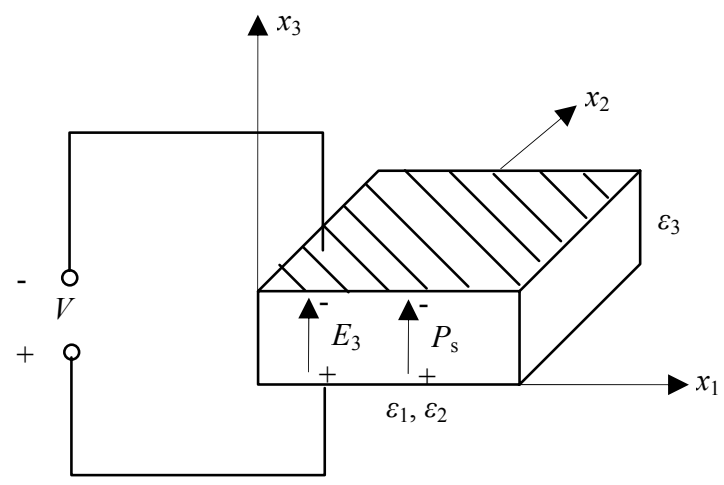

Figure 1 Induced strain response of PWAS

\subsection{Electro-Mechanical (E/M) Impedance Method}

The structural impedance method is a damage detection technique complementary to the wave propagation techniques. Ultrasonic equipment manufacturers offer, as options, mechanical impedance analysis (MIA) probes and equipment [12]. The mechanical impedance method consists of exciting vibrations of bonded plates using a specialized transducer that simultaneously measures the applied normal force and the induced velocity. Cawley [13] extended Lange's work on the mechanical impedance method and studied the identification of local disbonds in bonded plates using a small shaker. Though phase information was not used in Cawley's analysis, present day MIA methodology uses both magnitude and phase information to detect damage. The electro- 
mechanical (E/M) impedance method is an emerging technology [14] that uses in-plane strain excitation at the structural surface to measure the pointwise mechanical impedance of the structure in the form of the real part of the electrical impedance at the sensor terminals. The principles of the E/M impedance technique are illustrated in Figure 2 .

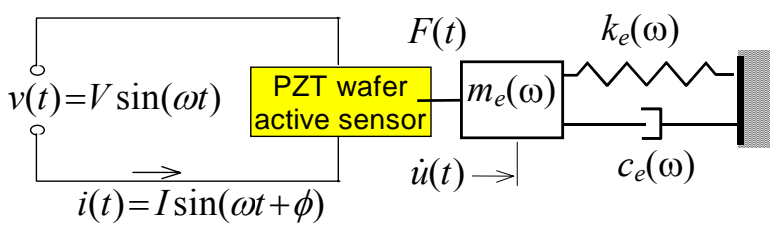

Figure 2 Electro-mechanical coupling between the PWAS and the structure

The effect of a piezoelectric active sensor affixed to the structure is to apply a local strain parallel to the surface that creates stationary elastic waves in the structure. The drive-point impedance presented by the structure to the active sensor can be expressed as a frequency-dependent quantity, $Z_{s t r}(\omega)=j \omega m_{3}(\omega)+c_{e}(\omega)-j k_{e}(\omega) / \omega$. Through the mechanical coupling between the PZT active sensor and the host structure, on one hand, and through the electro-mechanical transduction inside the PZT active sensor, on the other hand, the drivepoint structural impedance gets expressed in the effective electrical impedance, $Z(\omega)$, as seen at the active sensor terminals

$$
Z(\omega)=\frac{1}{j \omega c}\left[1-\kappa_{31}^{2}\left(1-\frac{1}{\varphi \cot \varphi+r(\omega)}\right)\right]^{-1}
$$

where $C$ is the zero-load capacitance of PWAS, $\kappa_{31}$ is the electro-mechanical cross-coupling coefficient $\left(\kappa_{31}=d_{13} / \sqrt{s_{11} \varepsilon_{33}}\right), r(\omega)$ is the impedance ratio between the pointwise structural impedance, $Z_{s t r}(\omega)$, and the impedance of PWAS, $Z_{\mathrm{PWAS}}$, and $\varphi=1 / 2 \gamma_{a} l_{a}$, with $\gamma_{a}$ being the active sensor wave number and $l_{a}$ its linear dimension. The electro-mechanical impedance method is applied by scanning a predetermined frequency range in the high $\mathrm{kHz}$ band and recording the complex impedance spectrum. By comparing the impedance spectra taken at various times during the service life of a structure, meaningful information can be extracted pertinent to structural degradation and the appearance of incipient damage. (It must be noted that the frequency range must be high enough for the signal wavelength to be significantly smaller than the defect size.)

\subsection{Guided Plate Waves (Lamb Waves) Method}

Lamb waves (a.k.a., guided plate waves) are a type of ultrasonic waves that are guided inside thin plates [15]. Lamb waves can propagate in a number of modes, either symmetrical or antisymmetrical. The velocity depends on the product of frequency and material thickness. Investigations on Lamb and leaky Lamb waves have been carried out continuously since their discovery, and researchers have done theoretical and experimental work for different purposes, ranging from seismology, to ship construction industry, to acoustic microscopy, and to nondestructive testing and acoustic sensors [16]-[18]. Keilers and Chang [19] and Chang [20] used piezoelectric-wafers to detect damage-detection in composite plates. The Lamb wave speed is obtained by solving the Rayleigh-Lamb equation. First, define $\xi=\sqrt{c_{S}^{2} / c_{P}^{2}}, \zeta=\sqrt{c_{S}^{2} / c_{L}^{2}}$, and $d=k_{\mathrm{S}} d$; where $c_{\mathrm{L}}$ is the Lamb wave speed, and $d$ is the half-thickness of the plate. In addition, define Lamb wave number $k_{\mathrm{L}}=\omega / c_{\mathrm{L}}$, and the variables $q=\sqrt{k_{L}^{2}-k_{P}^{2}}, s=\sqrt{k_{L}^{2}-k_{S}^{2}}$. Then for symmetric wave motion (Figure 3a), the Rayleigh-Lamb frequency equation can be written as

$$
\frac{\tan \left(\sqrt{1-\zeta^{2} d}\right)}{\tan \left(\sqrt{\xi^{2}-\zeta^{2}}\right)}+\frac{4 \zeta^{2} \sqrt{1-\zeta^{2}} \sqrt{\xi^{2}-\zeta^{2}}}{\left(2 \zeta^{2}-1\right)^{2}}=0
$$

The two components of the particle movement can be written as

$$
U(x, z, t)=\operatorname{Re}\left[A k_{L}\left(\frac{\cosh (q z)}{\sinh (q d)}-\frac{2 q s}{k_{L}^{2}+s^{2}} \frac{\cosh (s z)}{\sinh (s d)}\right) e^{j\left(k_{L} x-\omega t(\pi / 2)\right)}\right]
$$




$$
W(x, z, t)=\operatorname{Re}\left[A q\left(\frac{\sinh (q z)}{\sinh (q d)}-\frac{2 k_{L}^{2}}{k_{L}^{2}+s^{2}} \frac{\sinh (s z)}{\sinh (s d)}\right) e^{j\left(k_{L} x-\omega t\right)}\right]
$$

For antisymmetric motion (Figure 3b), the Rayleigh-Lamb frequency equation is

$$
\frac{\tan \left(\sqrt{1-\zeta^{2} d}\right)}{\tan \left(\sqrt{\xi^{2}-\zeta^{2}}\right)}+\frac{\left(2 \zeta^{2}-1\right)^{2}}{4 \zeta^{2} \sqrt{1-\zeta^{2}} \sqrt{\xi^{2}-\zeta^{2}}}=0
$$

And the two components of the particle movement can be written as

$$
\begin{gathered}
U(x, z, t)=\operatorname{Re}\left[A k_{L}\left(\frac{\cosh (q z)}{\cosh (q d)}-\frac{2 q s}{k_{L}^{2}+s^{2}} \frac{\cosh (s z)}{\cosh (s d)}\right) e^{j\left(k_{L} x-\omega t(\pi / 2)\right)}\right] \\
W(x, z, t)=\operatorname{Re}\left[A q\left(\frac{\sinh (q z)}{\cosh (q d)}-\frac{2 k_{L}^{2}}{k_{L}^{2}+s^{2}} \frac{\sinh (s z)}{\cosh (s d)}\right) e^{j\left(k_{L} x-\omega t\right)}\right]
\end{gathered}
$$

Figure 4 presents the dispersive (frequency-dependent) symmetric (S0) and antisymmetric (A0) Lamb wave speeds in a 1.6-mm thick aluminum plate.

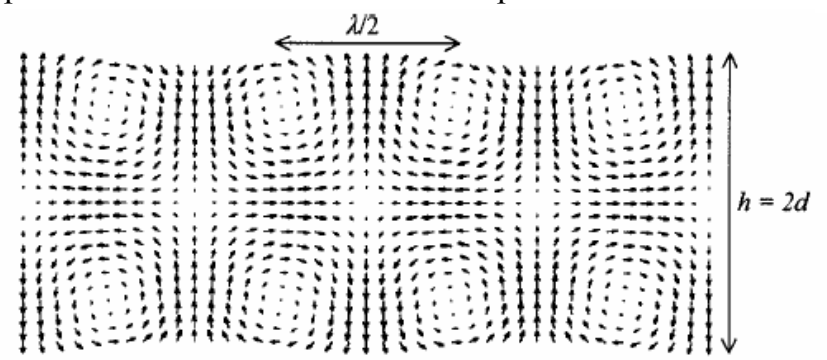

(a)

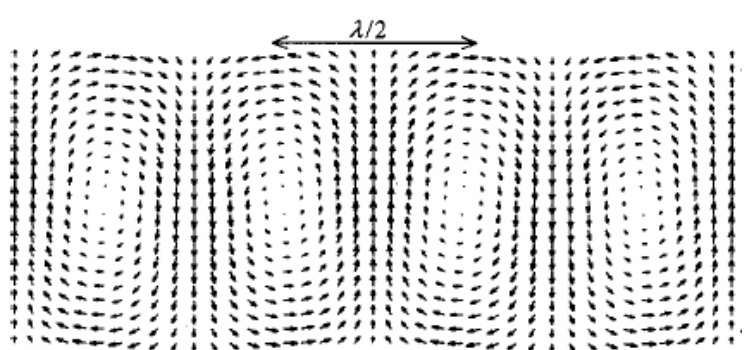

(b)

Figure 3 Simulation of Lamb waves in a 1-mm thick aluminum plate. (a) symmetric mode S0 at $f=1.56 \mathrm{MHz}$; (b) antisymmetric mode $\mathrm{A} 0$ at $f=0.788 \mathrm{MHz}$.
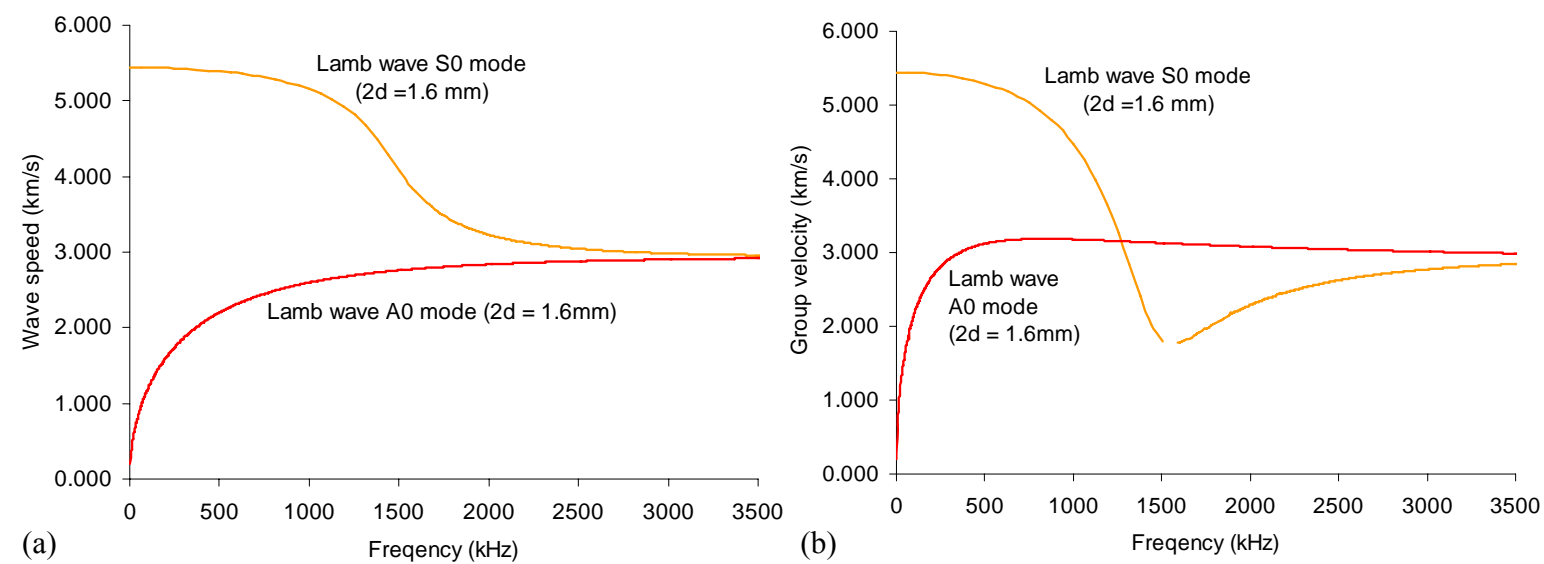

Figure 4 Dispersion curves for S0 and A0 Lamb wave modes in aluminum structure. (a) wave speed; (b) group velocity

\section{MULTI-MODE SENSING FOR PIPE CORROSION DETECTION}

The PWAS multi-mode sensing system will take advantage of PWAS ability: (1) to serve as modal sensor measuring structural impedance spectrum, (2) to serve as guided wave transducer in Lamb wave application, and (3) to measure the wall thickness. Once a PWAS sensing system, i.e., PWAS sensor network, is established on the structure, it will detect the corrosion defect in the structure by using the three methods simultaneously by altering driving frequencies. The detection procedure consists of three steps:

1. Lamb waves inspection using pitch catch method. The data will be analyzed by a damage index method. The pitch-catch pair which is suffering the largest change will be considered as having corrosion on 
that path.

2. Once the path is determined, E/M impedance spectrum will be obtained for all the PWAS along the path and analyzed by damage index method. The transducer which has the largest change will be determined as the one on the corrosion.

3. Finally, direct thickness measurements will be conducted to quantify the thinning. By using this strategy, the corrosion area will be located and measured.

\subsection{Damage index analysis}

When guided waves travel through a region where there is a change in material properties, such as the decrease of thickness in corrosion, the directly transmitted signal is modified and the change of receiving signal can provide good information about the corrosion development across the transmitting and receiving path. One way to represent the change is to subtract the baseline data for the structure without damage from the receiving data for the structure with the corrosion damage. The difference is a good indicator for the corrosion detection parameter since it carries information of both amplitude and the phase changes from the growth of the corrosion. The damage index (DI) is therefore defined as the relative ratio of the difference between each measurement and baseline signals to the baseline signal

$$
\text { Damage index (DI) }=\sqrt{\frac{\sum_{j=0}^{N-1}\left[s_{i}(j)-s_{0}(j)\right]^{2}}{\sum_{j=0}^{N-1} s_{0}^{2}(j)}}
$$

Where $s_{i}$ is the $i^{\text {th }}$ measurement and $s_{0}$ is the baseline signal, and $N$ is the data length. The DI equation will also be used to evaluate the changes in $\mathrm{E} / \mathrm{M}$ impedance spectrum to determine the impedance changes.

\subsection{PWAS thickness measurement}

PWAS can also work in thickness mode using $d_{33}$ effect. Experiments have been conducted using the setup shown in Figure 5a to measure plate thickness with PWAS.

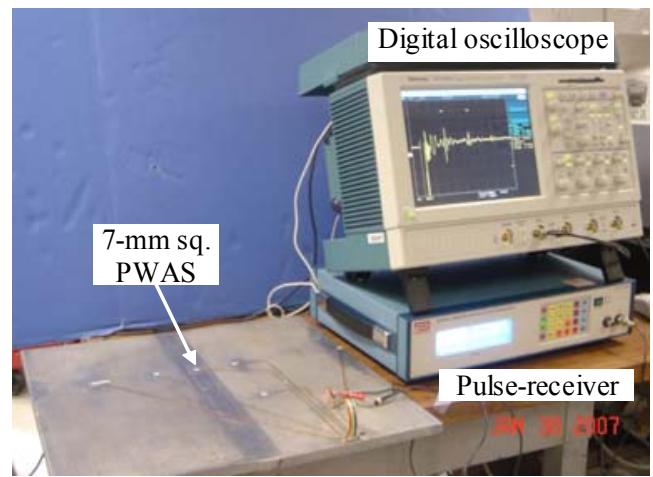

(a)

Figure 5 PWAS thickness measurement. (a) laboratory experiment setup; (b) pulse-echo signal collected using a 7-mm square PWAS on a $13.5-\mathrm{mm}$ thick steel plate at room temperature $(c=5850 \mathrm{~m} / \mathrm{s})$

A plate specimen was installed with a square or round PWAS on one surface of the plate. The PWAS were connected to a digital pulse/echo device (Panametrics 5900PR). Under electric excitation, PWAS generates an elastic wave pulse that spreads out into the plate. When the wave pulse hits the other surface, it is reflected back as an echo to the PWAS and then picked up by the digital oscilloscope synchronized with the pulse/echo device. A typical pulse-echo signal is shown in Figure $5 \mathrm{~b}$, which indicates a delay of $\Delta t$ between the initial pulse and the first reflected echo. The echo strength is strongest at first, which is the first reflected wave from the lower surface, and becomes weaker for the later echoes. Given the wave speed $c$ of the specified material, the thickness $T$ can be calculated as

$$
T=\frac{\Delta t \cdot c}{2}
$$




\section{MULTI-MODE CORROSION DETECTION EXPERIMENTS}

The PWAS network was installed on an aluminum pipe of $36.9 \mathrm{~mm}$ outside diameter and of $1.70 \mathrm{~mm}$ thickness (Figure 6a). The network uses five 7-mm, round PWAS as shown in Figure $6 \mathrm{~b}$. The corrosion damage underneath PWAS \#2 is simulated by materials loss through chemical corrosion at room temperature. Pitchcatch experiment sends out a 3-count tone-burst signal from a HP 33120A signal generator to one PWAS and the received signal at another PWAS is recorded by a TDS-210 digital oscilloscope. Two pristine signals collected at PWAS \#4 when PWAS \#0 is excited at two different frequencies, $57 \mathrm{kHz}$ and $165 \mathrm{kHz}$, are shown in Figure 5c and Figure 5d, respectively. It can be seen that PWAS can indeed generate and receive guided waves with good quality on pipe structure. It is further noticed with the increase of driving frequency, a new wave packet (as indicated in Figure 5d) shows up before the second wave packet (as indicated in both Figure 6c and Figure 6d).

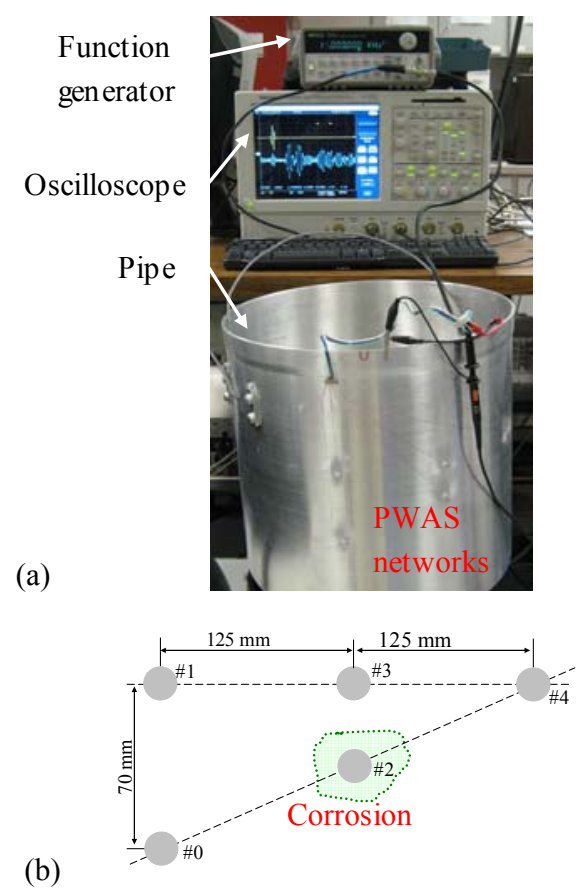

(c)
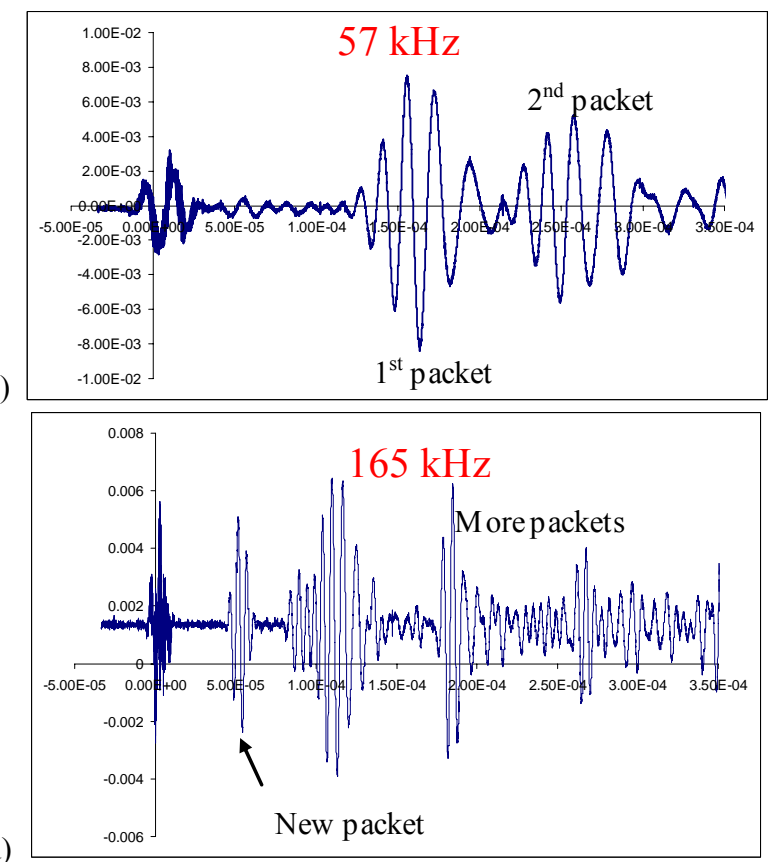

Figure 6 PWAS multi-mode sensing on aluminum pipe. (a) experimental setup; (b) schematic of a 5-PWAS network; (c) signal collected on \#4 when \#0 transmits at $57 \mathrm{kHz}$; (d) signal collected on \#4 when \#0 transmits at $165 \mathrm{kHz}$

\subsection{Corrosion detection on plate}

The sensing was first performed on metallic plate to detect and quantify the gradual corrosion at room temperature. The aluminum specimen is a $500 \mathrm{~mm} \times 500 \mathrm{~mm} \times 3.229 \mathrm{~mm}$ rectangular plate. Clay was place on the border to damp out boundary reflections. The 5-PWAS network shown in Figure $6 \mathrm{~b}$ was installed on the plate. Experimental setup is identical to pipe test unless otherwise specified. A $5 \mathrm{~mm}$ x $3.8 \mathrm{~mm}$ area underneath PWAS \#2 on the other side of the plate simulates the corrosion by removing material, as shown in Figure 7. Pitch-catch experiments were conducted on the PWAS pairs 1-4 and 0-4 (both have \#4 receiving). The corrosion on path 0-4 was recognized using damage index (DI) analysis first. Then impedance spectra were recorded and compared for PWAS on the path to locate PWAS \#2, which is the closest to the corrosion site. Finally PWAS \#2 measured the local thickness. Gradual thickness change is listed in Table 1.

Table 1 Thickness changes on aluminum plate 


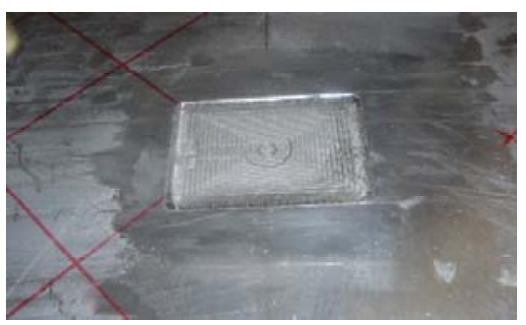

\begin{tabular}{|c|c|c|}
\hline Records & $\Delta d_{i}(\mathrm{~mm})$ & $\Delta d_{i} / d_{0}(\%)$ \\
\hline 0 & 0 & 0 \\
\hline 1 & 0.381 & 11.81 \\
\hline 2 & 0.7366 & 22.81 \\
\hline 3 & 0.9398 & 29.1 \\
\hline 4 & 1.09728 & 33.98 \\
\hline 5 & 1.27 & 39.33 \\
\hline
\end{tabular}

Figure 7 Simulated corrosion on aluminum plate.

\subsubsection{Pitch-catch and DI analysis}

The first step was the pitch-catch and DI analysis on all PWAS pairs. Signals received on PWAS \#4 on pair 0-4 at $120 \mathrm{kHz}$ at various corroded depth are shown in Figure 8a. The first arriving wave packet (faster) is S0 mode while the second packet (slower) is the A0 mode. DI curves of A0 mode and S0 mode are plotted in Figure 8b, respectively. It shows that $\mathrm{S} 0$ is not affected by the corrosion degradation while A0 mode clearly indicates the wall thinning on the path. We conclude that $\mathrm{A} 0 \mathrm{Lamb}$ wave mode is good for corrosion detection. DI curve obtained from pair 1-4 using A0 mode is also presented in Figure 8c, in comparison to the DI of pair 0-4 using A0 mode. Since the corrosion is away from the path 1-4, the DI is not significantly affected by the growth of corrosion as that of pair $0-4$. Hence, by comparing the DI curves, it is determined that the corrosion is located on the path 0-4 where largest DI change takes place.

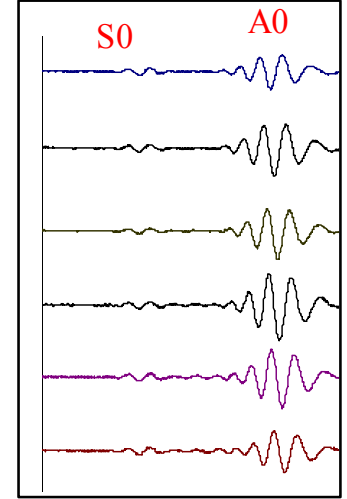

(a)

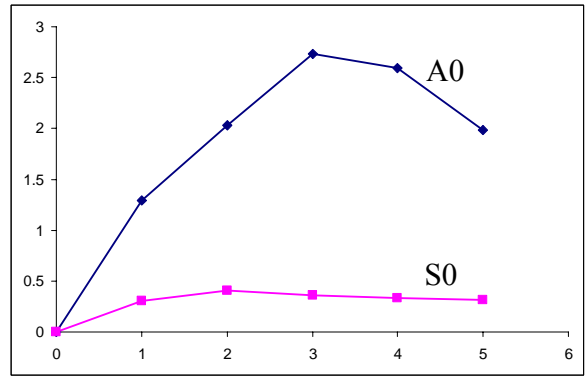

(b)

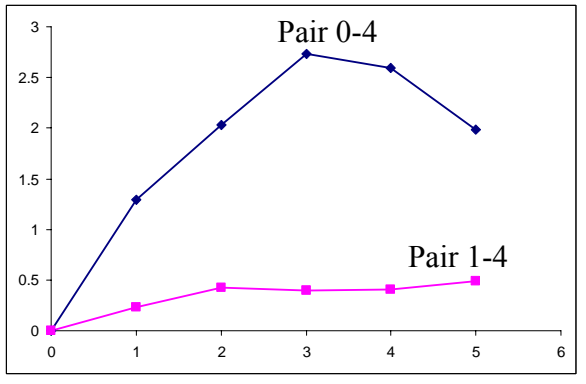

(c)

Figure 8 DI analysis on pitch-catch signals for corrosion detection. (a) received signal at PWAS \#4 when PWAS \#0 sent at 120 $\mathrm{kHz}$; (b) DI curves of both A0 and S0 wave packets at $120 \mathrm{kHz}$ of path 0-4; (c) DI curves of path 0-4 and path 1-4, comparing their sensitivity to corrosion damage

\subsection{2. $\quad$ E/M impedance spectrum}

After path 0-4 was identified as the corroded path, impedance measurement was used to locate the corrosion position. Impedance spectra of PWAS \#0, \#2, and \#3 were recorded by HP 4194A impedance analyzer over the frequency band $200 \sim 2000 \mathrm{kHz}$ where several fundamental modes were observed, given in Figure 9a c. (Note that \#4 was omitted since it is symmetric with $\# 0$ about $\# 2$ and \#3 was intentionally investigated in order to show the difference between \#2 and \#3). The spectrum difference between "baseline" data and "corroded" data can be quantified by processing the data, the real part of the E/M impedance spectra, with DI method. The RMSD DI curves are shown in Figure 9d. From the curves it is found that PWAS \#2 was undergoing the maximum changes while PWAS \#0 and PWAS \#3 barely changed with corrosion. 
(a)

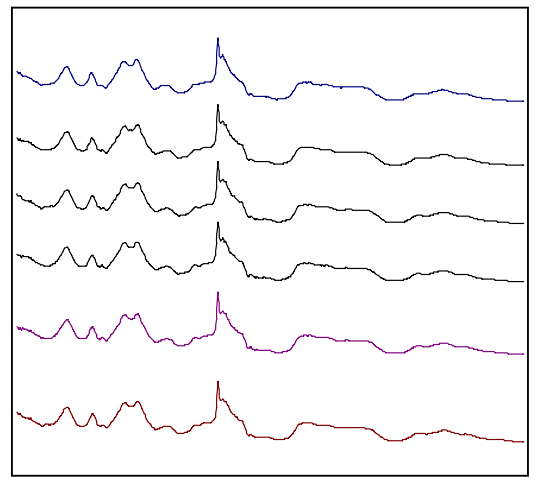

PWAS \#0

(c)

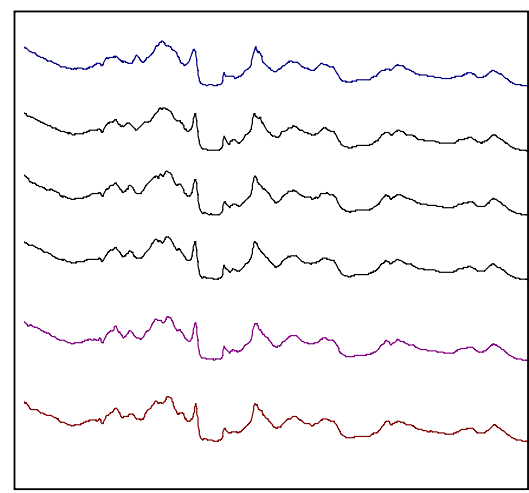

PWAS \#3 (b)

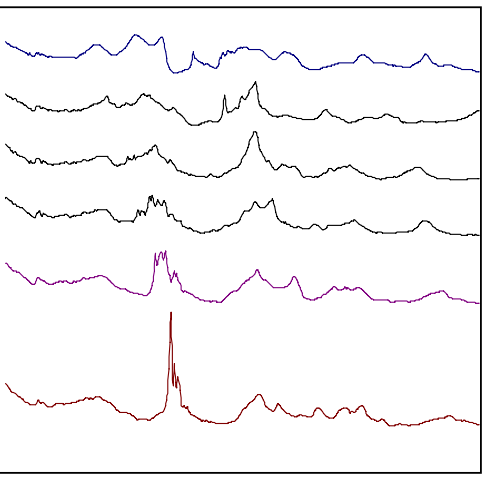

PWAS \#2

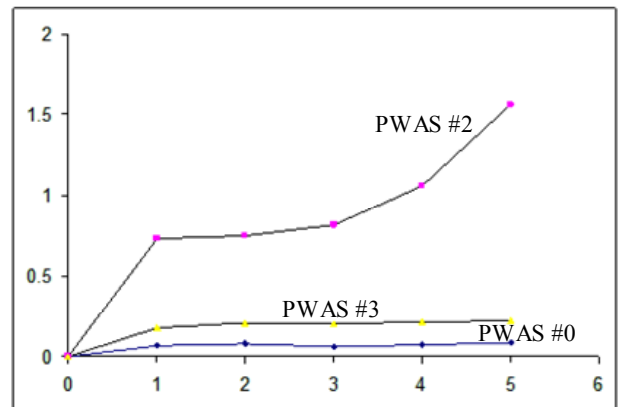

(d)

Figure 9 Impedance spectrum and DI curves. (a) real spectra of PWAS \#0; (b) real spectra of PWAS \#2; (c) real spectra of PWAS \#3 (d) DI curves

\subsubsection{Thickness measurement}

Once PWAS \#2 (location of corrosion) was identified, it is used to directly measure the thickness at its position. To compare the performance of PWAS thickness measurement, a $30 \mathrm{MHz}$ conventional ultrasonic transducer was used in parallel for comparison. The measurement results are given in Table 2.

Table 2 Thickness measurement using PWAS \#2 and ultrasonic transducer

\begin{tabular}{|c|c|c|c|c|c|}
\hline Records & $T_{0}, \mathrm{~mm}$ & $T_{\text {PWAS }}, \mathrm{mm}$ & $\varepsilon_{\text {PWAS }}, \%$ & $T_{\text {ultrasonic }}, \mathrm{mm}$ & $\varepsilon_{\text {ultrasonic }}, \%$ \\
\hline 0 & 3.229 & 3.354 & 3.87 & 3.069 & 4.96 \\
\hline 1 & 2.848 & 2.9072 & 2.08 & 2.7022 & 5.12 \\
\hline 2 & 2.4924 & 2.5034 & 4.41 & 2.3481 & 5.78 \\
\hline 3 & 2.2892 & 2.298 & 3.84 & 2.174 & 5.03 \\
\hline 4 & 2.137 & 2.218 & 3.79 & 2.062 & 3.50 \\
\hline 5 & 1.959 & 2.1742 & 10.98 & 1.839 & 6.13 \\
\hline
\end{tabular}

$T_{0}$-theoretical thickness, $c$-wave velocity, $6.212 \mathrm{~mm} / \mu \mathrm{s}$

Thickness measuring results show that PWAS provides reasonable results despite their low cost. Subsequent work is needed to investigate error ranges of both conventional transducers and PWAS. A high performance thickness gauge is to be considered and further investigation on the error ranges of the two transducers is to be conducted.

\subsection{Corrosion detection on pipe}

The corrosion detection was repeated on pipe as shown in Figure 6. Chemical corrosion was implemented using hydrochloric acid (37\% ACS) for 45 minutes processing. The resulting corrosion is not exactly under PWAS \#2. The corrosion process was carried for 11 times until completely failure as shown in Figure 10a. The setup and parameters are the same as those used in plate testing unless otherwise specified. 


\subsubsection{Pitch-catch and DI analysis}

Pitch-catch was tested at the selected frequency, $57 \mathrm{kHz}$ where A0 mode dominates. Signals received on PWAS \#4 in pair 1-4 and pair 0-4 were processed with the RMSD DI, respectively. The resulting curves, as shown in Figure 10b immediately shows larger changes on pair 0-4, indicating that the path from PWAS \#0 to PWAS \#4 was suffering corrosion damage. When compare the DI curves of pair 0-4 to that in Figure $6 \mathrm{~b}$, the same DI evolution is observed: the curve first increases rapidly and then decreases. The peak in pipe corrosion detection shows up at about half thickness being corroded.

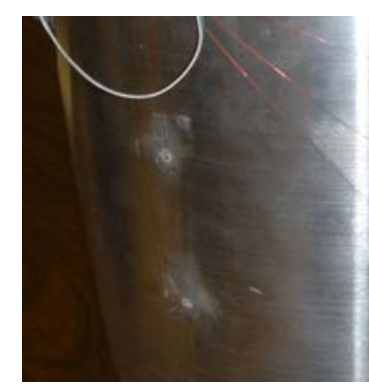

(a)

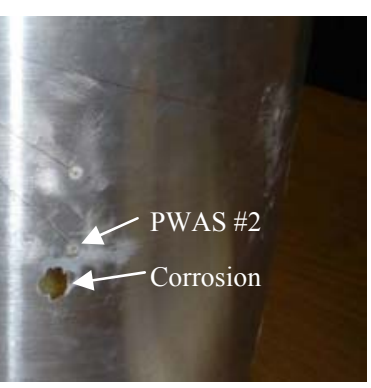

(b)

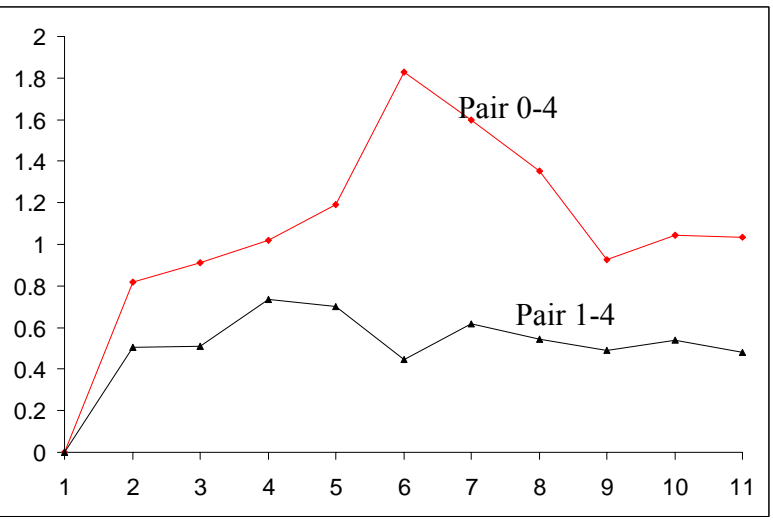

Figure 10 Corrosion on pipe. (a) corrosion damage details; (b) DI curves of path 0-4 and path 1-4

\subsection{2. $\quad$ E/M impedance spectrum}

Impedance spectra of PWAS \#0, \#2, and \#3 on the pipe were recorded by HP 4194A impedance analyzer over the frequency band 200 2000 kHz. The change of the real part spectrum is evaluated by the DI algorithm, as shown in Figure 11. From the curves PWAS \#2 was found undergoing the maximum changes while PWAS \#0 and PWAS \#3 barely changed with corrosion. Note that the readings 2,3,4 of PWAS \#0 were lost for bad wiring.

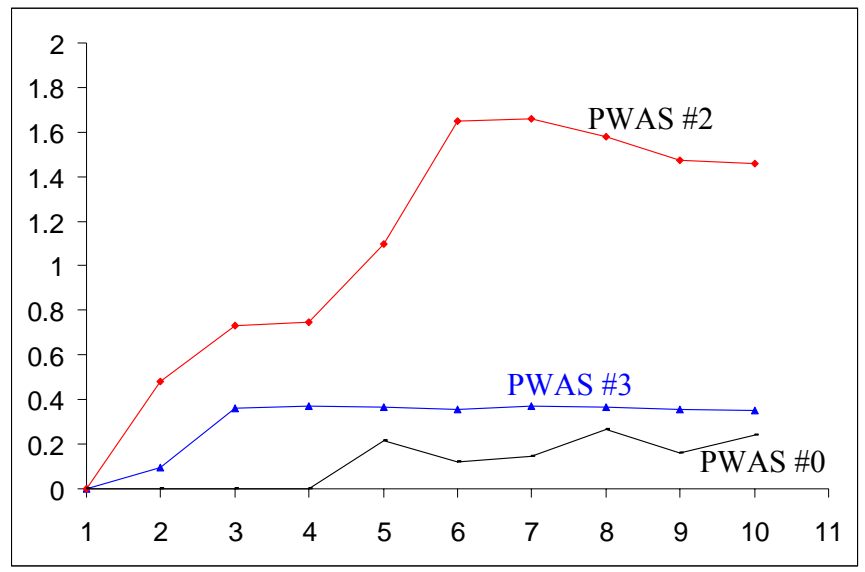

Figure 11 Pipe corrosion detection impedance spectrum DI curves

\subsubsection{Thickness measurement}

The original pipe thickness is $1.57 \mathrm{~mm}$ which is smaller than the minimum value investigated in the thickness measurement tests (see Table 2). Hence, the reading from the PWAS measurement is hard to be processed to find $\Delta t$. The thickness measurement has not been conducted in the pipe test. Miniaturized PWAS are expected to be used for small thickness measurement in the future.

\section{CONCLUSIONS}

Structural health monitoring with piezoelectric wafer active sensors (PWAS) used in this paper can interrogate 
structures through guided waves traveling at large distances into plate and shell structures, or detect local changes as modal sensors, or measure the thickness like the ultrasonic thickness gauge. The objective of our research is to create a multi-mode sensing methodology and technology that can permit the installation of an insitu structural health-monitoring network and perform all methods to provide increased detection ability. When applied to pipeline industries, it will improve the diagnostic efficiency and eliminate the regular shutdowns for preventive inspection and maintenance, thus considerably reducing the operation costs, decreasing personnel exposure, and increasing the safety of critical pipelines.

After a brief introduction of the principle of PWAS, E/M impedance measurement, and PWAS Lamb wave, a detailed description of the proposed the PWAS multi-mode sensing method was given. Extensive laboratory experiments were presented to verify the corrosion detection efficiency for both plate and pipe specimens at room temperature. The multiple sensing uses the pitch-catch method to find out the sensor path where corrosion is occurring and impedance spectrum to locate the sensor closest to the corrosion. Damage index (DI) diagnosis method was used to quantify the change in pitch-catch and impedance measurements. It has been noticed that when the plate was suffering thinning, the DI curve increased at first while when the thickness decreased to about $40 \%$ of the original thickness, the curve decreased instead. Due to the localization property of impedance spectrum, the DI curve of the impedance spectra shows that only the sensor undergoing corrosion changes significantly while the sensor near the corrosion does not change that much. After the corrosion position is found, the current thickness was measured to quantify changes. The thickness measurement using PWAS in plate tests shows good consistence and the performance is comparable to the conventional ultrasonic transducer. However, if the thickness is small, e.g. $1.70 \mathrm{~mm}$ in the pipe test, PWAS was unable to obtain reliable readings. Further investigation concerning PWAS thickness gauge ability needs to be considered and the minimum measurable thickness needs to be determined as well.

Currently, the effect of using different types of PWAS (e.g., square or round PWAS, decreased size PWAS) for the accuracy of the measured results is underway. The dependence of changes in signals on the material, geometry (e.g. curved or flat surface), and environment will be further investigated both theoretically and experimentally. As founded in this paper, S0 mode wave will not be affected by the corrosion defect while A0 mode changes significantly with corrosion development. Experiments on using different modes to detect different defects will be conducted to further investigate this potential of embedded PWAS guided waves monitoring.

\section{ACKNOWLEDGMENTS}

The financial support of National Science Foundation under Grant \#CMS-0408578 is thankfully acknowledged.

\section{REFERENCES}

[1] Krautkramer NDT (1998) “Emerging Technology - Guided Wave Ultrasonics”, NDTnet, Vol. 3, No. 6, June 1998

[2] Harker, A. H. (1988) Elastic Waves in Solids, British Gas, UK

[3] Chahbaz, A.; Mustafa, V.; Hay, D. R. (1996) "Corrosion Detection in Aircraft Structures using Guided Lamb Waves", ASNT Fall Conference 1996, NDTet, Vol. 1, No. 11, November 1996, http://www.ndt.net/article/tektrend/tektrend.htm

[4] Chahbaz A., Gauthier J.; Brassard, M.; Hay, D. R. (1999) "Itrasonic Technique for Hidden Corrosion Detection in Aircraft Wing Skin", Proceedings of the $3^{\text {rd }}$ Joint Conference on Aging Aircraft, 1999

[5] Alleyne, D. N.; Cawley P. (1992) “Optimization of Lamb Wave Inspection Techniques”, NDT\&E International, Vol. 25, No. 1, pp. 11-22

[6] Zhu, W.; Rose, J.L.; Agarwala, V.S. (1999) "Experimental Study on Hidden Corrosion/Delamination Detection with Ultrasonic Guided Waves", Defense Technical Information Center, Accession \# ADD344128

[7] Giurgiutiu, V., and Zagrai, A. (2002) "Embedded Self-Sensing Piezoelectric Active Sensors for On-Line Structural Identification,"' ASME J. Vibr. Acoust., Vol.124, Jan., pp. 116-125

[8] Bartkowicz, T. J., Kim, H. M., Zimmerman, D. C., and Weaver-Smith, S. (1996), "Autonomous Structural Health Monitoring System: A Demonstration," Proc., $37^{\text {th }}$ AIAA/ASME/ASCE/AHS/ASC Structures, Structural Dynamics, and Materials Conference, Salt Lake City, UT, April 15-17

[9] Boller, C., Biemans, C., Staszewski, W., Worden, K., and Tomlinson, G. (1999) “'Structural Damage Monitoring Based on an Actuator-Sensor System,', Proc., SPIE Smart Structures and Integrated Systems Conference, Newport, CA. March 1-4

[10] Boller, C. (2001) “Ways and Options for Aircraft Structural Health Monitoring,” Smart Mater. Struct., Vol.10, pp. $432-440$

[11] Giurgiutiu, V. (2007) Structural Health Monitoring with Piezoelectric Wafer Active Sensors, Elsevier, Burlington, MA

[12] Staveley NDT Technologies, “Sonic Bondmaster ${ }^{\text {TM }}$ Product Description,', Kennewick, WA 99336 
[13] Cawley, P. (1984) “'The Impedance Method for Non-Destructive Inspection,'” NDT Int., Vol. 17, No. 2, pp. 59-65

[14] Giurgiutiu, V., and Zagrai, A. (2001) “Electro-Mechanical Impedance Method for Crack Detection in Metallic Plates," SPIE's $8^{\text {th }}$ Annual International Symposium on Smart Structures and Materials, March 4-8, Newport Beach, CA, Paper No. 4335-22

[15] Viktorov, I. A. (1967) Rayleigh and Lamb Waves, Plenum Press, New York, NY

[16] Krautkramer, J., and Krautkramer, H. (1990) Ultrasonic Testing of Materials, Springer-Verlag

[17] Rose, J. L. (1999) Ultrasonic Waves in Solid Media, Cambridge University Press

[18] Lemistre, M., Gouyon, R., Kaczmarek, H., and Balageas, D. (1999) "Damage Localization in Composite Plates Using Wavelet Transform Processing on Lamb Wave Signals," $2^{\text {nd }}$ International Workshop of Structural Health Monitoring, Stanford University, September 8-10, pp. 861-870

[19] Keilers, C. H., and Chang, F.-K. (1995) "Identifying Delaminations in Composite Beams Using Built-in Piezoelectrics: Part I-Experiments and Analysis; Part II An Identification Method,' J. Intell. Mater. Syst. Struct., Vol.6, Sept., pp. 649-672

[20] Chang, F.-K. (1998) "Manufacturing and Design of Built-in Diagnostics for Composite Structures," $52^{\text {nd }}$ Meeting of Society for Machinery Failure Prevention Technology, Virginia Beach, VA, March 30_April 3 